\title{
Perioperative management of coexistent fat oxidation defect and malignant hyperthermia
}

\section{S.S. Nair ${ }^{1}$, J. Paul ${ }^{1}$ \\ McMaster University, Hamilton, Canada}

\section{Background}

Multiple acyl-CoA dehydrogenase deficiency (MADD) is a rare autosomal recessive error of metabolism. Also known as glutaric aciduria type 2 (GA2), it is an autosomal recessive inborn error of metabolism affecting the oxidation of fatty acids. MADD is caused by deficiency of either an electron-transfer flavoprotein (ETF) or an electron-transfer flavoprotein dehydrogenase (ETFDH).

\section{Case presentation}

A 28 year old male presented for elective first stage urethroplasty with bilateral buccal grafts.

He had had bilateral ureteric implantation aged 9. A diagnosis of malignant hyperthermia was made following his surgery. There were repeated hospital presentations during childhood with generalised weakness. He was then investigated for fat oxidation defects. The 3 hydroxy 3 methyl glutaric acid, heptenedioic acid and palmitic acid were raised. His carnitine palmitoyltransferase I (CPT 1), carnitine palmitoyltransferase 2(CPT2), short chain acyl-coenzyme $A$ dehydrogenase (SCAD) and long chain acyl-coenzyme $A$ dehydrogenase (LCAD) were normal and a diagnosis of MADD was made. His echocardiogram at the time was normal. Over time his symptoms subsided.

On the day of surgery, he was started on a $10 \%$ dextrose infusion running at $150 \mathrm{ml} /$ hour as soon as he was admitted. Standard monitors were applied. Anesthesia was induced with $2 \mathrm{mg}$ of midazolam, $180 \mathrm{mg}$ of propofol at $2.5 \mathrm{mg} / \mathrm{kg}$ and $50 \mathrm{mg}$ of rocuronium at $0.6 \mathrm{mg} / \mathrm{kg}$. A remifetanil infusion was started at $0.1 \mathrm{mcg} / \mathrm{kg} / \mathrm{min}$ and increased to $0.2 \mathrm{mcg} / \mathrm{kg} / \mathrm{min}$. Anesthesia was maintained with propofol at $120 \mathrm{mcg} / \mathrm{kg} / \mathrm{min}$ and reduced to $100 \mathrm{mcg} / \mathrm{kg} / \mathrm{min}$ after an hour. A right radial arterial line was inserted. During the case, serial arterial blood gases, blood glucose and creatine kinase levels were measured and were within normal limits. At the end of the procedure, patient was extubated and transferred to intensive care. His postoperative period was uneventful and he was discharged after 5 days.

\section{Discussion}

Acyl-CoA dehydrogenases are essential for fatty acid oxidation. MADD results in decreased activity of acyl-CoA dehydrogenases involved in electron transfer from acyl-CoA dehydrogenase to coenzyme $Q$ in the mitochondrial electron transport chain resulting in inhibition of mitochondrial oxidation of fatty acids ${ }^{1 .}$

The clinical features of patients with MADD fall into 3 classes: a neonatal-onset form with congenital anomalies (type I), a neonatalonset form without congenital anomalies (type II), and a late-onset form (type III).

Types I and II are characterized by severe nonketotic hypoglycemia, metabolic acidosis and multiorgan involvement along with the excretion of large amounts of fatty acid- and amino acid-derived metabolites. Type III is characterized by recurrent episodes of lethargy, vomiting, hypoglycemia, metabolic acidosis, and hepatomegaly. These episodes are precipitated by metabolic stress such as infection, low-energy diet, pregnancy or surgery. Metabolic derangements include elevated transaminases, raised creatine kinase, elevated serum levels of free fatty acids and hyperammonemia.

In our case, the co-existence of malignant hyperthermia along with MADD complicated anesthetic management as we had to avoid inhalational agents but at the same time use propofol in a patient with impaired beta-oxidation of fatty acids. The mitochondrial electron transport chain can be affected by propofol. Patients with mitochondrial disorders and carnitine deficiency are at a higher risk of developing propofol infusion syndrome? ${ }^{2}$.

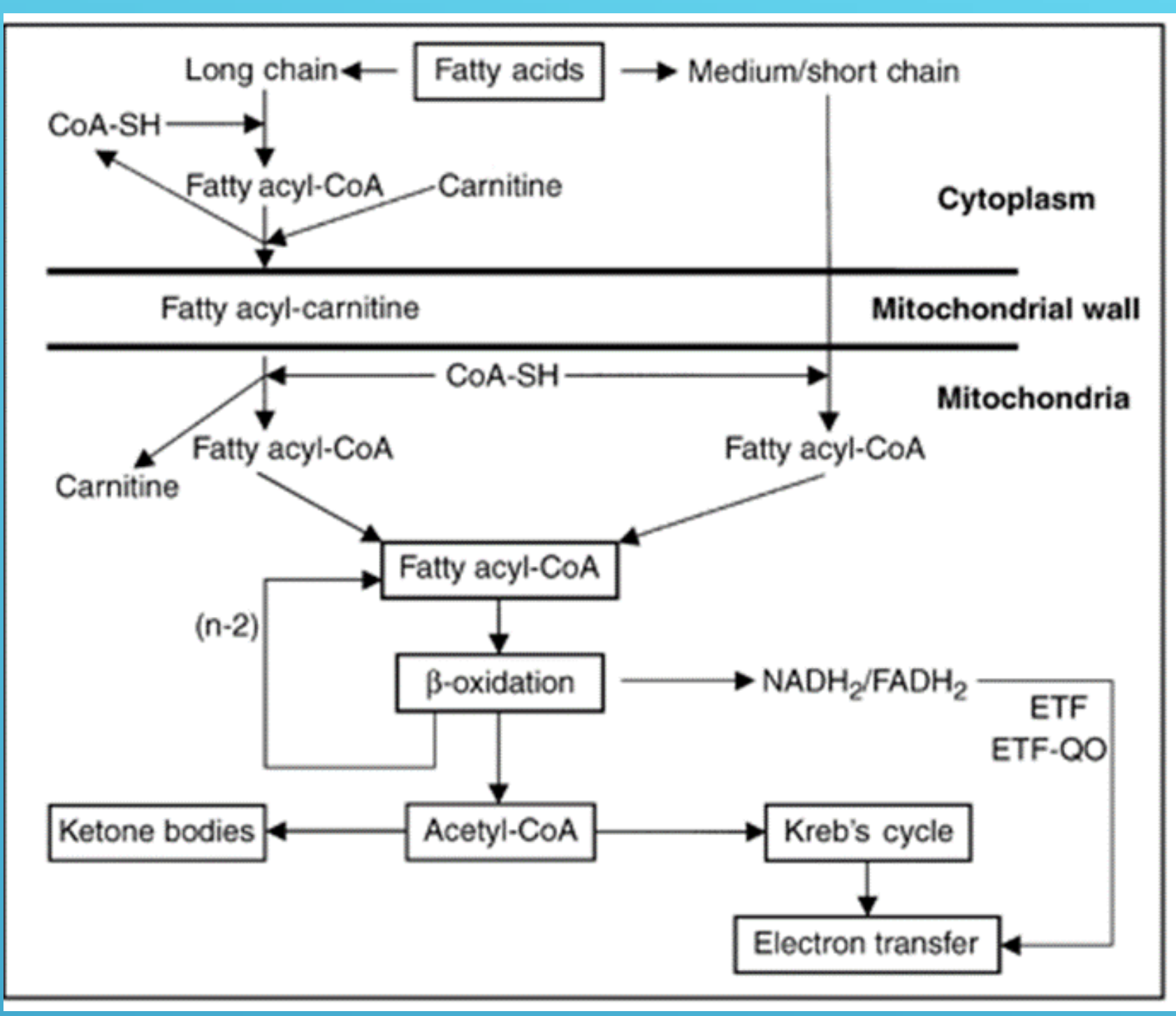

(From Grice AS, Peck TE. (2001). Multiple acyl-CoA dehydrogenase deficiency: a rare cause of acidosis with an increased anion gap. British journal of Anaesthesia, 86(3), 437-441.)

Maintaining normoglycemia is one of the vital perioperative concerns as this patient population is prone to impaired glucose metabolism and are unable to utilize fatty acids ${ }^{3}$. Normothermia needs to be maintained as temperature regulation requires energy derived from oxidative metabolism.

\section{Conclusion}

Our case report highlights the important anesthetic considerations for patients with mitochondrial disease and concurrent malignant hyperthermia. These patients are rare but at the same time we should be aware of the potential multi-organ dysfunctions that they can present with in the perioperative period. This case report reiterates anesthetics considerations such as normothermia, normoglycaemia and minimizing metabolic stress in this patient population. It also emphasizes the potential neurologic and metabolic disturbances that these patients can develop.

\section{References}

1.Olsen RK, Olpin SE, Andresen BS, Miedzybrodzka ZH, Pourfarzam M, Merinero B, et al . ETFDH mutations as a cause of riboflavin-responsive multiple acyl-CoA dehydro deficiency. Brain 2007; 130:2045-54. 2.Parness J, Savard M, Turgeon AF. Propofol-related infusion syndrome heralding a mitochondrial disease: case report. Neurology 2014;82(5):461-462. 3.Farag E, Argalious M, Narouze S, DeBoer GE, Tome J.The anesthetic management of ventricular septal defect (VSD) repair in a child with mitochondrial cytopathy. Can J Anaesth. 2002;49(9):958-962. 